\title{
Changes in Quantitative Profile of Extracellular Matrix Components in the Kidneys of Rats with Adriamycin-induced Nephropathy
}

\author{
Noboru MANABE ${ }^{1)}$, Akiko KINOSHITA ${ }^{1)}$, Misuzu YAMAGUCHI ${ }^{1)}$, Yoshihiro FURUYA ${ }^{1)}$, Nobuo NAGANO ${ }^{2}$, \\ Kozue YAMADA-UCHIO ${ }^{1)}$, Naotsugu AKASHI ${ }^{1)}$, Keiko MIYAMOTO-KURAMITSU ${ }^{1)}$ and Hajime MIYAMOTO ${ }^{1)}$ \\ 1) Unit of Anatomy and Cell Biology, Department of Animal Sciences, Kyoto University, Kyoto 606-8502, and ${ }^{2)}$ Pharmaceutical Research \\ Laboratory, Kirin Brewery Co., Ltd., Takasaki 370-1202, Japan
}

(Received 2 August 2000/Accepted 28 September 2000)

ABSTRACT. Extracellular matrix components (ECMs) in histological sections of the kidney cortex from the rats with adriamycin (ADR)induced nephropathy $(5 \mathrm{mg} / \mathrm{kg}$, i.v.) were quantified by an immunohistochemical micromethod. Changes in kidney histopathology and urine and blood biochemistry were investigated. Enlarged kidneys were granular on the surface and pale in color in ADR-treated rats, and these rats had kidneys with glomeruli with expanded mesangial area and with capillary aneurysm. Severe albuminuria, hypoalbuminemia, hypercholesterolemia and disorders in other nephrotic parameters were observed in ADR-treated rats. Type I and IV collagens, fibronectin and laminin contents in the renal cortex of ADR-treated rats at 10 weeks were 329,317, 263 and $295 \%$, respectively, higher than in each vehicle control, and those at 28 weeks were 1,211, 930, 1,057 and 1,012\%, respectively. The glomerular sclerotic abnormalities progressed in a time-dependent manner. Moreover, there was a strong correlation between the ECM levels and serum creatinine and blood urea nitrogen levels. In conclusion, microquantification provided useful information for accurate diagnosis and prognosis of nephrotic lesions and is a good tool to assess the advancement of renal disorders in patients with nephropathy.

KEY WORDS: adriamycin-induced nephropathy, extracellular matrix, glomerular sclerosis, immunohistochemical microquantification, rat.

J. Vet. Med. Sci. 63(2): 125-133, 2001

Adriamycin (ADR) is a commonly used antineoplastic antibiotic. In experimental animals, ADR has nephrotoxic actions and produces chronic progressive glomerular disease $[2,15,16]$. In rats with ADR-induced nephropathy, heavy proteinuria associated with loss of glomerular polyanion, focal fusion of foot processes and swelling as well as vacuolation of epithelial cells have been reported in shortterm experiments $[1,24]$. Severe renal damage, extensive glomerular lesions, tubular dilatation and stromal fibrosis have been observed in long-term studies $[5,15]$. These experiments indicated that ADR-induced nephropathy has chronic and self-perpetuating, leading to terminal renal failure in chronic renal diseases.

As the accumulation of extracellular matrix (ECM) components in the kidney cortex, mainly in the glomerulus, plays an important etiological role in chronic progressive nephropathy [9], histopathological evaluation of ECM accumulation in kidney biopsy sections is essential to render an accurate diagnosis. However, conventional histopathological estimation is not quantitative, and conventional biochemical methods available for measuring the ECM content require large tissue samples (at least $100 \mathrm{mg}$ of wet tissue) and are not sensitive enough for precise diagnosis [4, 10, 17]. Previously, we developed a new immunohistochemical micromethod for accurate quantification of ECMs in histological sections of normal rat kidneys [13, 14]. This micromethod is based on the enzyme-linked immunosorbent assay (ELISA), and frozen tissue sections mounted on glass slides are used as models of the antigen-precoated wells of ELISA plates [13, 14]. The micromethod is sensitive enough for quantification of ECMs in very small tissue samples such as biopsies, and makes it easy to quantitatively and objectively evaluate the ECM accumulation in histologic sections. Moreover, the tissue structure is well preserved during measurement in this micromethod.

The aim of the present study was to evaluate the usefulness of the microquantification method for histopathological diagnosis of renal failure using sections of the kidney cortex from rats with the experimental nephropathy induced by ADR injection.

\section{MATERIALS AND METHODS}

Animals and tissue preparation: Forty eight male Lewis rats (8-week-old) purchased from Charles River Japan (Kanagawa, Japan) were used. Nephropathy was induced by intravenous injection of ADR (Sigma Chemical Co., St. Louis, MO, U.S.A.) at a dose of $5 \mathrm{mg} / \mathrm{kg}$ body weight. In the vehicle control group, isotonic saline was given instead of ADR. After injection, the rats were kept for 10 or 28 days with access to tap water and a standard diet ad libitum in an air conditioned room $\left(22 \pm 1^{\circ} \mathrm{C}\right.$ and $\left.50 \pm 5 \%\right)$ under a $12 \mathrm{hr}$ light/dark schedule. All animals received humane care as outlined in the "Guide for the Care and Use of Laboratory Animals" (Kyoto University Animal Care Committee according to NIH \#86-23; revised 1999). Eight rats in each group were sacrificed on week 0,10 or 28 after injection. For clinical biochemical analyses, urine samples during the $24 \mathrm{hr}$ before sacrifice ( $24 \mathrm{hr}$ urine samples) were collected, and blood samples were obtained from the cervical vein under ether anesthesia. The animals were sacrificed under deep ether anesthesia, and then the kidneys were rapidly removed. One kidney was immediately fixed in $10 \%$ neutral-buffered formalin ( $\mathrm{pH}$ 7.4) for conventional histopatho- 
logical evaluation, while the other was frozen in dry iceisopentane mixture for immunohistochemical measurement of ECMs.

Clinical biochemistry: As previously reported [20, 22, 23], $24 \mathrm{hr}$ urine and serum samples were examined on the basis of the following biochemical parameters to evaluate nephrotic state. Urinary and serum albumin (uAlb and sAlb, respectively) levels were measured by a bromo-cresol green method using an A/GB test kit (Wako Pure Chemical Co., Osaka, Japan). Serum creatinine (sCr) and blood urea nitrogen (BUN) levels were measured by Jaffé's method with a creatinine test kit (Wako) and by the urease indophenol method with a nitrogen-B test kit (Wako), respectively. The serum total cholesterol (sTC) level was determined by an enzymatic method with a Cholesterol-E test kit (Wako).

Histopathology: After formalin fixation, the kidney samples were dehydrated through a graded ethanol series and embedded in Histosec (Merck Co., Darmstadt, Germany). Sections $3 \mu \mathrm{m}$ thick were prepared on a microtome, mounted on glass slides precoated with 3-aminopropyltriethoxysilane (Aldrich Chemical, Milwaukee, WI, U.S.A.), deparaffinized with xylene and rehydrated through a graded ethanol series. For conventional histopathological evaluation, some of the sections were stained with hematoxylin and eosin, or periodic acid-Schiff reagent (PAS) for alteration of basement membrane. The extent of glomerulosclerosis was expressed as the degree of collagen deposition, which was assessed on sections stained with Sirius red F3B solution (saturated picric acid in distilled water containing $0.1 \%$ Sirius red F3B; BDH Chemicals Ltd., Poole, UK) [12]. All slides were mounted with Entellan (Merck) and examined by light microscopy; at least three sections were examined per rat. A semiquantative score was determined to evaluate the degree of glomerular sclerosis, according to Okuda et al. [15]. Briefly, histopathological evaluation was made independently by two pathologists without prior knowledge of the experimental groups. In each kidney specimen, approximately 50 glomeruli in cortical sections selected at random and juxtamedullary portions were examined. Each glomerulus was classified into two categories: normal (glomerulus without mesangial expansion) and lesion (expansion of mesangial area, capillary aneurysm and/or hypercellularity). The severity of the lesion was graded from - (0) to $++++(4)$ according to the percentage of glomerular sclerosis; + (1), ++ (2), +++ (3) and $++++(4)$ indicated the involvement of $25,50,75$ and $100 \%$ of the glomerulus, respectively. An injury score was obtained by multiplying the degree ( 0 to 4 ) by the percentage of the glomeruli with the same degree of injury (increase in mesangial material or glomerular sclerosis). The extent of the injury for each tissue specimen was then obtained by the addition of these scores. For instance, 5 of 20 glomeruli showed (1 degree of injury) and 5 of 20 glomeruli showed ( 3 degree of injury), the injury score of this kidney would be: $((1 \times 5 / 20)+(3 \times 5 / 20)) \times 100=100$. In addition, the morphological changes in the glomeruli (capillary aneurysm and hypercellularity), and tubular (cystic tubular dilation, epithelial cellular atrophy and intraluminal cast formation) and tubulointerstitial (tubulointerstitial expansion and mononuclear cell filtration around arterioles) lesions were recorded.

Histochemical quantification of ECM: The degree of ECM deposition in kidney sections is a good indicator of glomerular sclerosis. Type I and IV collagens, fibronectin and laminin levels in each frozen section of rat kidney cortex were measured by a microquantification method as previously described [12-14, 20, 22, 23]. Briefly, after preincubation with $10 \mathrm{mM} \mathrm{NaN}_{3}$ and $100 \mathrm{mM} \mathrm{H}_{2} \mathrm{O}_{2}$ diluted with phosphate buffered saline containing 3\% bovine serum albumin (PBS-BSA) to inhibit the endogenous peroxidase activity, the slide was incubated with the appropriate first antibody; rabbit anti-rat type I collagen, IV collagen, fibronectin and laminin polyclonal antibodies purchased from Chemicon (Temecula, CA, U.S.A.), Chemicon, Sigma and ICN Pharmaceutical (Aurora, OH, U.S.A.), respectively, were diluted $1 / 100$ with PBS-BSA. After washing with PBS containing $0.05 \%$ Tween 20 (PBS-Tw), the slides were incubated with second antibody (horseradish peroxidase-conjugated goat anti-rabbit IgG antibody, 1/400 dilution with PBS-BSA) purchased from American Qualex (La Mirada, CA, U.S.A.). They were washed with PBS-Tw, each section ( $400 \mu \mathrm{l} /$ section) was incubated with chromogenic substrate solution $(30 \mathrm{mM}$ phenol, $3 \mathrm{mM} \mathrm{4-aminoan-}$ tipyrine and $2 \mathrm{mM} \mathrm{H}_{2} \mathrm{O}_{2}$ in $50 \mathrm{mM}$ Tris- $\mathrm{HCl}, \mathrm{pH}$ 7.2). Twenty $\mu l /$ section of $1 \mathrm{M} \mathrm{NaN}_{3}$ was added to stop the enzymatic reaction, and then the mixed solutions were transferred into microcuvettes to determine the optical density at $450 \mathrm{~nm}$ using a spectrophotometer (Ultrospec 3000, Pharmacia Biotech, Uppsala, Sweden). The ECM content in each section was calculated. After measurement, the total protein and total collagen levels in each section were measured colorimetrically [20]. In brief, each section was rinsed in a $0.6 \%$ acetic acid solution for $10 \mathrm{~min}$, washed with distilled water, and stained with $400 \mu l$ of the staining solution (a saturated solution of picric acid in distilled water containing $0.1 \%$ sirius red $\mathrm{F} 3 \mathrm{BA}$ and $0.1 \%$ fast green $\mathrm{FCF}$ ) for $2 \mathrm{hr}$. After 5 washes with distilled water, $1 \mathrm{ml}$ of elution medium (absolute methanol containing an equal volume of $0.1 \mathrm{~N} \mathrm{NaOH}$ ) was applied to each section, and then the slide was incubated for $30 \mathrm{~min}$. The elution medium was transferred to microcuvette, and the eluted color was read in the spectrophotometer at 540 and $630 \mathrm{~nm}$. A negative control section incubated without first antibody was processed in each run. Each measurement was performed in triplicate. All incubations were carried out in dark moist chambers.

Immunohistochemistry: Immunofluorescence staining for ECMs was performed as previously described [2, 23]. Briefly, frozen thin and thick sections (5 and $20 \mu \mathrm{m}$ thick) were cut on a cryostat for conventional and confocal immunofluorescence microscopy, respectively. The sections were mounted on 3-aminopropyltrimethoxysilane precoated slides, fixed with precooled acetone for $10 \mathrm{~min}$ at $-80^{\circ} \mathrm{C}$, and then incubated with the appropriate first antibody for 18 hr at $4^{\circ} \mathrm{C}$. They were washed with PBS, incubated with flu- 
orescein isothiocyanate-conjugated goat anti-rabbit IgG antibody (American Qualex), and then the thin and thick sections were examined under a fluorescence microscope (BX50, Olympus, Tokyo, Japan) and with a confocal laser scanning microscope (Fluoview FV300, Olympus), respectively.

Statistical analysis: ANOVA with Fisher's least significant differences test comparison for biochemical data, and Wilcoxon's signed rank test for morphological estimation were carried out with the Statview-IV program using a Macintosh computer. Differences at a probability of $\mathrm{P}<0.05$ were considered significant. All data are expressed as mean values $\pm \mathrm{SD}$.

\section{RESULTS}

Clinical parameters: Changes in body and kidney weights and clinical parameters are summarized in Table 1. Body weight was increased to a lesser extent in the ADRtreated rats than in controls $(372 \pm 13$ and $523 \pm 7 \mathrm{~g}$ at 28 weeks after ADR administration, respectively). In both groups, kidney weight (mean weight of right and left kidneys) increased. However, relative kidney weight decreased in controls, but increased in the ADR-treated rats $(0.27 \pm$ 0.02 and $0.46 \pm 0.04 \%$ at 28 weeks, respectively). In the control group, there were no significant differences in $\mathrm{uAlb}$, sAlb, sTC, sCr or BUN between any time point examined, but severe albuminuria, hypoalbuminemia, hypercholesterolemia and disorders in other nephrotic parameters were seen in the ADR-treated rats. The amount of uAlb progressively increased and reached levels of $1248 \pm 221$ and 1393 $\pm 318 \mathrm{mg} / \mathrm{day} / \mathrm{rat}$ at week 10 and 28, respectively. Significant decreases of sAlb (hypoalbuminemia; $2.80 \pm 0.33$ and $2.69 \pm 0.30 \mathrm{mg} / \mathrm{d} l$ ) with a marked increase in sTC (hypercholesterolemia; $377 \pm 71$ and $380 \pm 120 \mathrm{mg} / \mathrm{d} l$ ) were observed at week 10 and 28, respectively. At week 10, both BUN $(42 \pm 7 \mathrm{mg} / \mathrm{d} l)$ and $\mathrm{sCr}(1.12 \pm 0.56 \mathrm{mg} / \mathrm{d} l)$ began to increase, indicating severe loss of renal function. At week 28 , BUN and $\mathrm{sCr}(240 \pm 131$ and $3.55 \pm 1.31 \mathrm{mg} / \mathrm{d} l$, respectively) were increased significantly compared with controls (17 \pm 1 and $0.41 \pm 0.03 \mathrm{mg} / \mathrm{d} l$, respectively). Thus, the nephrotic state progressed in a time-dependent manner in the ADR-treated rats.
Renal histopathology and ECMs levels: In the ADRtreated rats, enlarged kidneys were granular on the surface and pale in color. The degree of glomerular sclerosis is summarized in Table 2. Conventional histopathological examination revealed that most control rats had kidneys with normal glomeruli $(3.8 \pm 0.7,4.6 \pm 1.2$ and $4.5 \pm 1.1$ degree at 0,10 and 28 weeks, respectively), but all ADRtreated rats had kidneys with glomeruli with expanded mesangial area and with capillary aneurysm $(4.1 \pm 0.6,54.1$ \pm 10.5 and $186.1 \pm 17.1$ degree at 0,10 and 28 weeks, respectively). Expansion of the mesangial areas with an apparent increase in the mesangial matrix, appearance of cysts, extension of renal tubules were observed in the kidney sections of ADR-treated rats (Fig. 1). Moreover, as compared with the kidney sections of ADR-treated rats at week 0 (Fig. 2A), progressed fibrotic change in the glomeruli was observed in the kidney sections of ADR-treated rats at 10 and 28 weeks. Briefly, in rats without ADR-treatment, no immunohistochemical reaction for type I collagen was detected in normal glomeruli and weak reaction was observed in the tubulointerstitum (Fig. 2A), and trace deposit of fibronectin was seen in glomeruli and the interstitium (data not shown). In the kidneys of ADR-treated rats, however, abundant deposits of interstitial components (type I collagen and fibronectin) were noted in expanded glomeruli and tubulointerstitial lesions, and such abnormalities progressed in a time-dependent manner (Fig. 2B and C). In kidneys of rats before ADR-treatment, trace deposits of basal membrane components (type IV collagen and laminin) were observed in glomeruli and the interstitium (data not shown). Deposit of type IV collagen progressively increased in the glomerular, especially expanded mesangial areas, and basement membranes of renal tubules, mainly extended tubules, of ADR-treated rat kidneys. In specimens stained for laminin, similar differences in immunofluorescence staining pattern were also observed between ADRtreated and control rats. Thus, glomerulonephritic and glomerular-fibrotic change became severe only in ADRtreated rats, and these histopathological abnormalities progressed in a time-dependent manner in the ADR-treated rats.

Renal ECM levels assessed by a microquantification method are also shown in Table 2. In control rats, no signif-

Table 1. Time courses of changes in body and kidney weight and clinical parameters in ADR-treated and control rats

\begin{tabular}{|c|c|c|c|c|c|c|}
\hline \multirow[t]{2}{*}{ Parameter } & \multicolumn{3}{|c|}{ Control rats ( $\mathrm{n}=8$, each point $)$} & \multicolumn{3}{|c|}{ ADR-treated rat ( $n=8$, each point) } \\
\hline & 0 & 10 & 28 & 0 & 10 & 28 (weeks) \\
\hline Body weight (g) & $308 \pm 3$ & $439 \pm 5 * *$ & $523 \pm 7 * *$ & $307 \pm 5$ & $384 \pm 12 * *$ & $372 \pm 13 * *$ \\
\hline Kidney weight (g) & $1.16 \pm 0.09$ & $1.30 \pm 0.09$ & $1.43 \pm 0.10$ & $1.15 \pm 0.09$ & $1.69 \pm 0.16^{* *}$ & $1.70 \pm 0.13^{* *}$ \\
\hline Relative kidney weight (\%) & $0.38 \pm 0.03$ & $0.30 \pm 0.02 * *$ & $0.27 \pm 0.02 * *$ & $0.37 \pm 0.03$ & $0.44 \pm 0.05^{* *}$ & $0.46 \pm 0.04 * *$ \\
\hline Urinary albumin (mg/day/rat) & $30 \pm 3$ & $29 \pm 3$ & $29 \pm 4$ & $28 \pm 4$ & $1,248 \pm 221 * * *$ & $1,393 \pm 318 * * *$ \\
\hline Serum albumin $(\mathrm{g} / \mathrm{d} l)$ & $4.14 \pm 0.07$ & $4.15 \pm 0.12$ & $4.18 \pm 0.10$ & $4.15 \pm 0.11$ & $2.80 \pm 0.33 * *$ & $2.69 \pm 0.30 * *$ \\
\hline Blood urea nitrogen $(\mathrm{mg} / \mathrm{d} l)$ & $17 \pm 2$ & $17 \pm 1$ & $17 \pm 1$ & $17 \pm 2$ & $42 \pm 7 * *$ & $240 \pm 131 * * *$ \\
\hline Serum cholesterol $(\mathrm{mg} / \mathrm{d} l)$ & $80 \pm 5$ & $78 \pm 5$ & $78 \pm 3$ & $78 \pm 8$ & $377 \pm 71 * *$ & $380 \pm 120 * *$ \\
\hline
\end{tabular}

$*, * *$ and ${ }^{* *}: \mathrm{P}<0.05,0.01$ and 0.001 vs respective control. 
Table 2. Extracellular matrix component levels in the kidneys of ADR-treated and control rats

\begin{tabular}{lccccccc}
\hline \multirow{2}{*}{ Extracellular matrix } & \multicolumn{3}{c}{ Control rats $(\mathrm{n}=8$, each point) } & & \multicolumn{3}{c}{ ADR-treated rats (n=8, each point) } \\
\cline { 2 - 3 } \cline { 6 - 7 } & 0 & 10 & 28 & & 0 & 10 & 28 (weeks) \\
\hline Glomerular sclerosis & $3.8 \pm 0.7$ & $4.6 \pm 1.2$ & $4.5 \pm 1.1$ & & $4.1 \pm 0.6$ & $54.1 \pm 10.5^{* *}$ & $186.1 \pm 17.4^{* * * *}$ \\
Type I collagen (mg/g) & $3.81 \pm 0.57$ & $3.71 \pm 0.52$ & $3.80 \pm 0.60$ & & $3.98 \pm 0.47$ & $12.21 \pm 3.09^{* *}$ & $46.01 \pm 19.88^{* * *}$ \\
Type IV collagen $(\mathrm{mg} / \mathrm{g})$ & $2.64 \pm 0.78$ & $2.09 \pm 0.72$ & $2.46 \pm 1.11$ & & $2.31 \pm 0.49$ & $6.63 \pm 2.25^{*}$ & $22.91 \pm 9.52^{* * *}$ \\
Fibronectin (mg/g) & $3.39 \pm 0.40$ & $3.39 \pm 0.78$ & $3.29 \pm 0.77$ & & $2.85 \pm 0.90$ & $8.90 \pm 2.58^{*}$ & $34.75 \pm 17.35^{* * *}$ \\
Laminin (mg/g) & $1.83 \pm 0.51$ & $1.73 \pm 0.54$ & $1.65 \pm 0.48$ & & $1.60 \pm 0.53$ & $5.09 \pm 1.73^{* *}$ & $16.70 \pm 6.47^{* * *}$ \\
\hline
\end{tabular}

Glomerular sclerosis: degree of glomerular sclerosis is a semiquantitative score as described in Materials and Methods. $*, * *$ and $* * *: \mathrm{P}<0.05,0.01$ and 0.001 vs respective control.

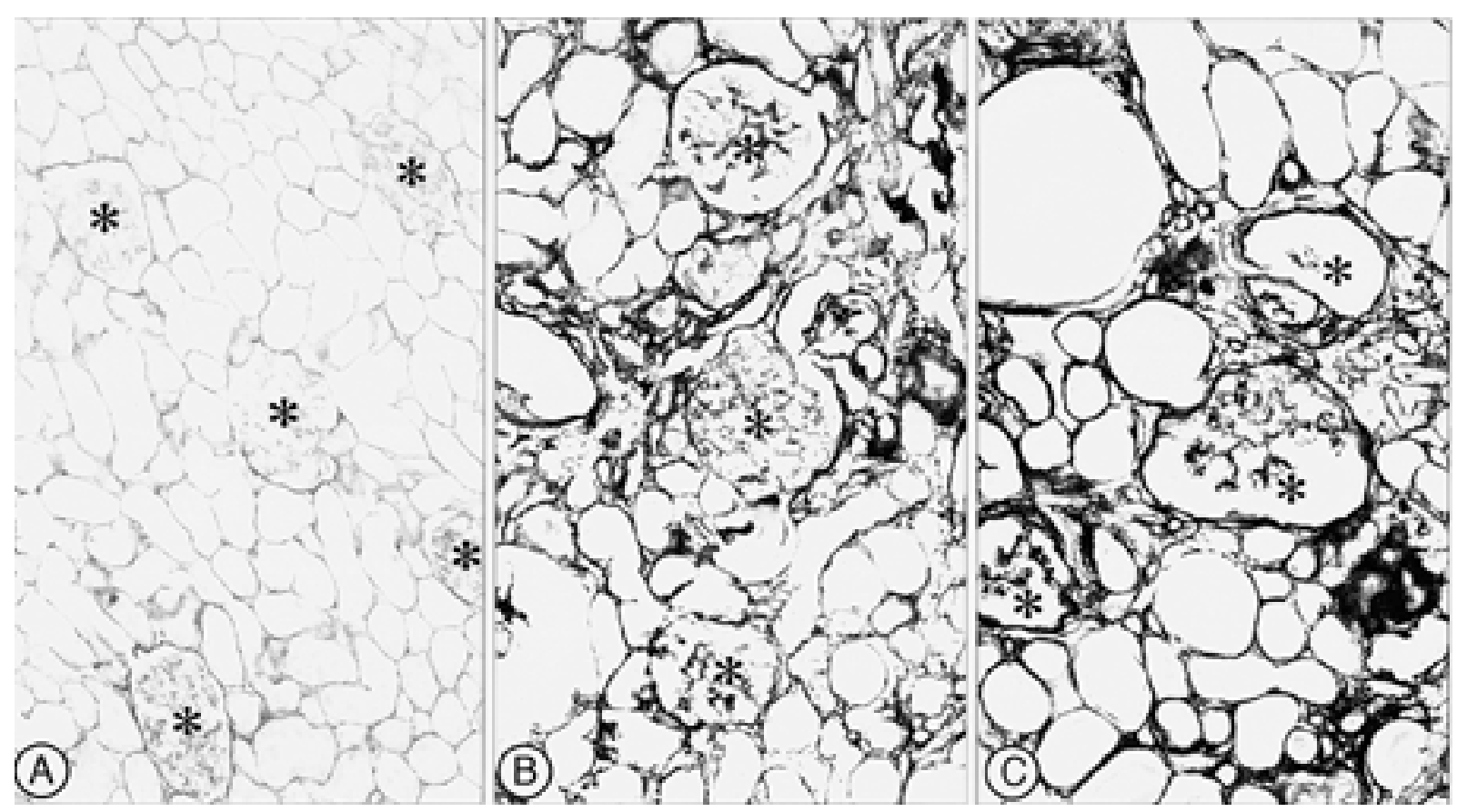

Fig. 1. Kidney sections of ADR-treated rats at 0, 10 and 28 weeks (A, B and C, respectively) were stained with Sirius red F3B solution. Severe fibrotic change in glomeruli and the basement membrane of renal tubules, appearance of cysts, extension of renal tubules and infiltration of inflammatory cells were observed in the kidney sections of ADR-treated rats at 10 and 28 weeks (B and C, respectively), but not in those at week 0 (A). Asterisks: glomeruli. $(\times 200)$

icant differences in type I collagen, type IV collagen, fibronectin or laminin levels in kidney cortex sections were seen among any time point examined. Type I and IV collagens, fibronectin and laminin contents in the renal cortex of ADR-treated rats at 10 weeks $(12.21 \pm 3.09,6.63 \pm 2.25$, $8.90 \pm 2.58$ and $5.09 \pm 1.73 \mathrm{mg} / \mathrm{g}$ of total protein, respectively) were $329,317,263$ and $295 \%$, respectively, higher than in the respective vehicle controls $(3.71 \pm 0.52,2.09 \pm$ $0.72,3.39 \pm 0.78$ and $1.73 \pm 0.54 \mathrm{mg} / \mathrm{g}$, respectively) $(\mathrm{P}<0.05$ in each case). These parameters of ADR-treated rats at 28 weeks $(46.01 \pm 19.88,22.91 \pm 9.52,34.75 \pm 17.35$ and $16.70 \pm 6.47 \mathrm{mg} / \mathrm{g}$, respectively) were $1,211,930,1,057$ and $1,012 \%$, respectively, higher than those in vehicle controls $(3.80 \pm 0.60,2.46 \pm 1.11,3.29 \pm 0.77$ and $1.65 \pm 0.48$ $\mathrm{mg} / \mathrm{g}$, respectively) $(\mathrm{P}<0.05$ in each case). Moreover, as compared with ADR-treated rats at week 0, those at 10 and 28 weeks showed 3.07-fold and 11.58-fold increases in type I collagen levels, 2.86-fold and 9.91-fold increases in type IV collagen levels, 3.12-fold and 12.19-fold increases in fibronectin levels, and 3.18-fold and 10.44-fold increases in laminin levels, respectively.

As shown in Fig. 3, significant correlations were found between kidney type I collagen levels and $\mathrm{sCr}$ and $\mathrm{BUN}$ levels $\left(r^{2}=0.996\right.$ and 0.954 , respectively) $(\mathrm{P}<0.001)$, but not uAlb, sAlb or sTC levels. These clinical biochemical parameters, $\mathrm{sCr}$ and BUN levels, showed significant correlations with the renal contents of type IV collagen $\left(\mathrm{r}^{2}=0.944\right.$ and 0.948 , respectively), fibronectin $\left(\mathrm{r}^{2}=0.931\right.$ and 0.974 , respectively) and laminin $\left(\mathrm{r}^{2}=0.950\right.$ and 0.934 respectively) $(\mathrm{P}<0.001)$ (Fig. 4). 

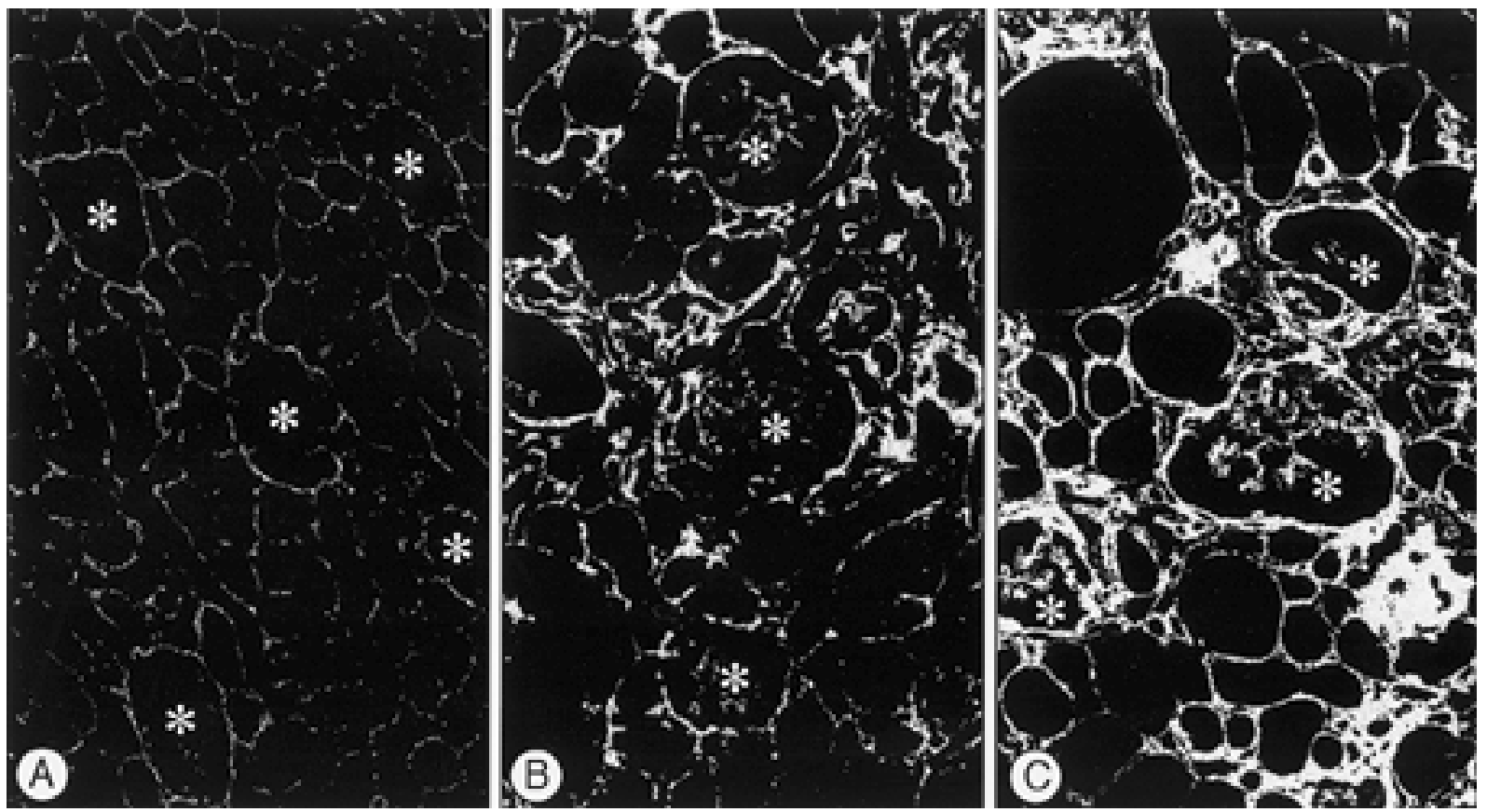

Fig. 2. Kidney sections prepared from ADR-treated rats at 0,10 and 28 weeks (A, B and C, respectively) immunohistochemically stained for type I collagen. In the sections of week 0 ADR-treated rats (A), no positive reactions for type I collagen were demonstrated in glomeruli. However, in the kidney sections of those at 10 and 28 weeks (B and C, respectively), strong positive reactions for type I collagen were seen in expanded glomeruli, and tubulointerstitial lesions. Glomerular-fibrotic change progressed in a time-dependent manner in the ADR-treated rats. Asterisks: glomeruli. $(\times 200)$

\section{DISCUSSION}

ADR caused various immunological and hemodynamic changes and induced chronic nephropathy [15]. The renal lesions induced by intravenous administration of ADR in rat kidneys were vacuolated changes in glomeruli at earlier stages, and either segmental or global sclerosis with tubulointerstitial changes at the progressed stage. The mechanism responsible for such renal pathological lesions induced by ADR administration remains unclear. However, several hypotheses regarding the pathogenic mechanism of ADR have been proposed. Lopez et al. [11] reported that ADR increased the lipoperoxide content but decreased the glutathione content in rat kidneys, and such oxidative stress causes tubulointerstitial lesions. Fernandez et al. [6] demonstrated that ADR-induced nephrotic syndrome is associated with marked decreases in expression of aquaporin-2, -3 and -4 , and the vasopressin-regulated urea transporter in the renal inner medulla, and the ADR causes suppression of the capacity for water and urea absorption by the inner medullary collecting duct and the extracellular volume expansion observed in ADR-nephrotic syndrome. Tamaki et al. [19] showed that overexpression of activated form of transforming growth factor (TGF)- $\beta 1$ might be related to glomerulosclerosis and interstitial fibrosis in ADR-nephropathy. Rats with ADR-induced nephropathy have been used to evaluate the therapeutic effects of many drugs. For example, capto- pril, an angiotensin converting enzyme inhibitor used for antihypertensive therapy, slowed down mesangial expansion and reduced the development of glomerular sclerosis in spontaneously hypertensive rats with ADR-induced nephropathy [8], indicating that antihypertensive therapy delays the progression of chronic renal failure. Melatonin, a pineal indole, has antioxidative activity, and retinol palmitate, an antioxidative reagent, both showed a protective effect against the hyperlipidemic nephropathy induced by ADR in rats [11], indicating that the antioxidative activity of these reagents neutralizes the damaging effect of ADR-induced free radicals in the kidney.

Earlier studies revealed that the most important histopathological feature of kidney diseases, fibrotic change, is caused by ECM accumulation in glomeruli and/or the tubulointerstitum $[3,7]$. In the present study, we confirmed that such fibrotic changes progress in a time-dependent manner in the kidneys of ADR-treated rats. We examined what types of ECM components are accumulated in the kidneys in this model and determined the correlation between the histopathological and clinical biochemical characteristics of the renal lesions of ADR-treated rats. Major ECM components, both interstitial (type I collagen and fibronectin) and basement membrane (type IV collagen and laminin) components, were found to be markedly accumulated in the renal cortex of ADR-treated rats. As compared with before ADR treatment, the rats at 10 and 28 weeks after ADR treatment 

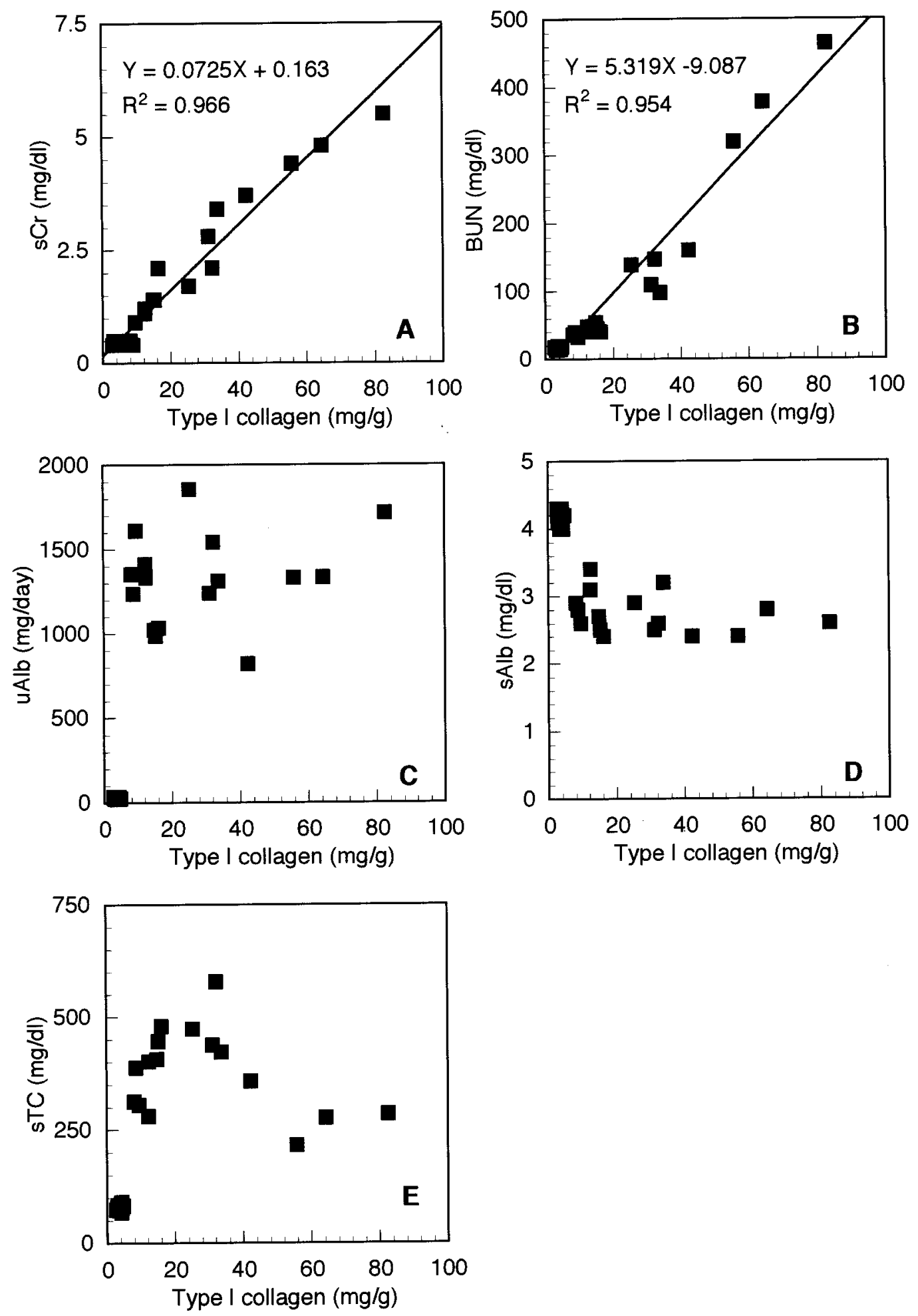

Fig. 3. Correlations between kidney type I collagen levels and clinical biochemical parameters (sCr, BUN, uAlb, sAlb and sTC shown in A, B, C, D and E, respectively). Significant correlations were seen with $\mathrm{sCr}$ and $\mathrm{BUN}(\mathrm{P}<0.001$; $\mathrm{r}^{2}=0.996$ and 0.954 , respectively), but not uAlb, sAlb or sTC. 

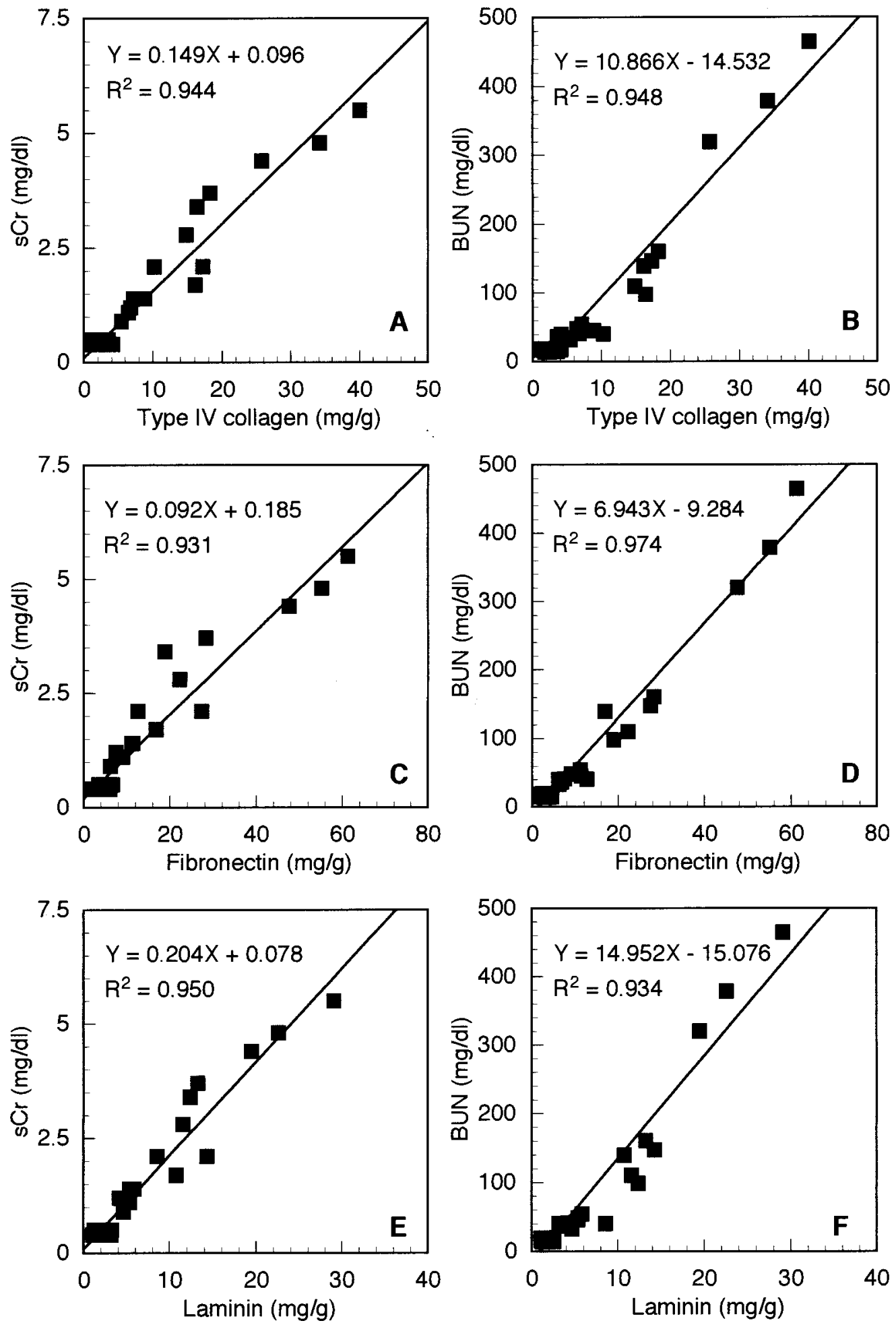

Fig. 4. Significant correlations between ECMs (type IV collagen, fibronectin and laminin) and clinical biochemical parameters ( $\mathrm{sCr}$ and BUN). $\mathrm{sCr}$ and BUN showed significant correlations with type IV collagen ( $\mathrm{A}$ and $\mathrm{B} ; \mathrm{r}^{2}=0.944$ and 0.948 , respectively), fibronectin $\left(\mathrm{C}\right.$ and $\mathrm{D} ; \mathrm{r}^{2}=0.931$ and 0.974 , respectively) and laminin ( $\mathrm{E}$ and $\mathrm{F} ; \mathrm{r}^{2}=0.950$ and 0.934 respectively) $(\mathrm{P}<0.001)$. 
showed 3.07-fold and 11.58-fold increases in type I collagen levels, 2.86-fold and 9.91-fold increases in type IV collagen levels, 3.12-fold and 12.19-fold increases in fibronectin levels, and 3.18-fold and 10.44-fold increases in laminin levels, respectively. There are two possible mechanisms of such deposition of ECM in the ADR-treated kidneys; ECM in the kidney tissues may be overproduced, or ECM breakdown may be inhibited in ADR-treated rats. The active form of TGF- $\beta 1$ up-regulates ECM production and down-regulates degradation of the ECM through its inhibitory effect on the matrix metalloproteinase that plays a key role in ECM degradation [18, 21-23]. Previously, we demonstrated that both possible mechanisms, i.e., acceleration of ECM synthesis and inhibition of ECM breakdown, are strongly related to TGF- $\beta 1$ in the kidneys with nephropathy of ICGN mice, a novel animal model of hereditary nephrotic syndrome [12, 20, 22, 23]. As mentioned above, a progressive increase in TGF- $\beta 1$ expression and activation in the renal cortex and glomeruli of ADR-treated rats was reported [19], indicating that TGF- $\beta 1$ promotes the pathological progression of renal fibrotic change characterized by ECM accumulation in ADR-treated rats. We demonstrated histopathologically that most glomerular mesangial cells in damaged glomeruli modulated into myofibroblast-like cells (immunopositive to $\alpha$-smooth muscle actin; data not shown) in the kidneys of ADR-treated rats, and such modulation may be caused by increased TGF- $\beta 1$. Thus, the pathogenetic process in the glomeruli of ADR-treated rat kidneys is presumed to be mediated by the increased levels of the active form of TGF- $\beta 1$, which would induce mesangial cell transformation, and these activated mesangial cells would then overproduce ECMs. Tubulointerstitial fibrotic change was also found in ADR-treated rats. We presume that the tubulointerstitial cells were induced to differentiate into fibroblast-like cells by activated TGF- $\beta 1$ in ADR-treated rats, and then the differentiated tubulointerstitial cells would synthesize and release excessive ECMs into the intercellular spaces between the renal tubules. Furthermore, severe albuminuria, hypoalbuminemia, hypercholesterolemia and disorders in other nephrotic parameters were observed in ADRtreated rats. There was a strong correlation between type I collagen level in the renal cortex assessed by a microquantification method, and $\mathrm{sCr}$ and BUN levels, but not $\mathrm{uAlb}$, sAlb or sTC level. The $\mathrm{sCr}$ and BUN levels also showed strong correlations between the renal levels of type IV collagen, fibronectin and laminin, indicating that excessive ECM deposition in the kidneys of ADR-treated rats is directly correlated with loss of renal function.

In conclusion, the micromethod described here is sensitive enough to measure the contents of ECM proteins in small sections of kidney biopsy samples, and makes it possible to quantify the ECM levels in biopsy sections. This microquantification technique provides useful information for accurate diagnosis and prognosis of nephrotic lesions as well as for estimation of therapeutic efficacy of drugs. Further studies are currently in progress to assess the chronological changes in ECM levels in this experimental model of nephropathy and to quantify the ECM levels in the biopsy sections from other experimental models, irreversible glomerulosclerosis induced by repeated injections of anti-Thy 1.1. antibody and glomerular and tubulointerstitial fibrosis in the hereditary nephrotic model, ICGN mice.

ACKNOWLEDGEMENTS. This work was supported partly by a grant-in-aid to N. M. and H. M. from the Ministry of Education, Science, Sports and Culture of Japan (05660344, 07660379, 11556052, 12480252). The authors wish to thank J.-A. Grimaud (Université Paris VI, France), and M. Chevallier and S. Guerret (Institut Pasteur, France) for valuable advice on the immunohistochemical microquantification technique.

\section{REFERENCES}

1. Bertani, T., Poggi, A., Pozzoni, R., Delani, F., Sacchi, F., Thoua, Y., Mecca, G., Remuzzi, G. and Donati, M. B. 1982. Adriamycin-induced nephrotic syndrome in rats: Sequence of pathologic events. Lab. Invest. 46: 16-23.

2. Bertazzoli, C., Chieli, T., Ferni, G., Ricevuti, F. G. and Solicia, E. 1972. Chronic toxicity of adriamycin: A new antineoplastic antibiotic. Toxicol. Appl. Pharmacol. 21: 287-301.

3. Couchman, J. R., Beavan, L. A. and McCarthy, K. J. 1994. Glomerular matrix: synthesis, turnover and role in mesangial expansion. Kidney Int. 45: 328-335.

4. Duhamel, R. C., Meezan, E. and Brendel, K. 1981. The addition of SDS to the Bradford dye-binding protein assay, a modification with increased sensitivity to collagen. J. Biochem. Biophys. Methods 5: 67-74.

5. Fajardo, L. F., Eltringham, J. R., Stewart, J. R. and Klauber, M. R. 1980. Adriamycin nephrotoxicity. Lab. Invest. 43: 242-253.

6. Fernandez, L. P., Andrews, P., Nielsen, S., Ecelbarger, C. A., and Knepper, M. A. 1998. Impaired aquaporin and urea transporter expression in rats with adriamycin-induced nephrotic syndrome. Kidney Int. 53: 1244-1253.

7. Floege, J., Johnson, R. J., Gordon, K., Iida, H., Pritzl, P., Yoshimura, A., Campbell C, Alpers, C. E. and Couser, W. G. 1991. Increased synthesis of extracellular matrix in mesangial proliferative nephritis. Kidney Int. 40: 477-488.

8. Jovanovic, D., Dimitrijevic, J., Varagic, J., Jovovic, D., Starcevic, A., and Djukanovic, L. 1998. Effects of captopril on morphologic changes in kidney of spontaneously hypertensive rats with adriamycin nephropathy. Renal Fail. 20: 451-458.

9. Kashgarian, M. and Sterzel, R. B. 1992. The pathobiology of the mesangium. Kidney Int. 41: 524-529.

10. Klasson, S. C., Klein, L. N., Dimari, S. J. and Haralson, M. A. 1986. The effects of tissue pretreatment and pepsin levels on the isolation of collagens from human placenta. Collagen Rel. Res. (Matrix) 6: 397-408.

11. Lopez, M. P., Finana, T. I., Munoz, D-A. C., Luna, G. F., and Lopez, S. J. V. 1998. Protective role of melatonin and retinol palmitate in oxidative stress and hyperlipidemic nephropathy induced by adriamycin in rats. J. Pineal Res. 25: 86-93.

12. Manabe, N., Chvallier, M., Chossegros, P., Causse, X., Guerret, S., Trépo, C. and Grimaud, J-A. 1993. Interferon-a2b therapy reduces liver fibrosis in chronic non-A, non-B hepatitis: a quantitative histological evaluation. Hepatology 18: 13441349.

13. Manabe, N., Furuya, Y., Nagano, N. and Miyamoto, H. 1994. 
Immunohistochemical microquantitation method for type I collagen in kidney histological section of the rats. J. Vet. Med. Sci. 56: $147-150$.

14. Manabe, N., Furuya, Y., Nagano, N., Yagi, M., Kuramitsu, K. and Miyamoto, H. 1995. Immunohistochemical quantitation for extracellular matrix proteins in rats with glomerulonephritis. Nephron 71: 79-86.

15. Okuda, S., Oh, Y., Tsuruda, H., Onoyama, K., Fijimi, S. and Fujishima, S. 1986. Adriamycin-induced nephropathy as a model of chronic progressive glomerular disease. Kidney Int. 29: 502-510.

16. Philips, F. S., Gilladoga, A., Marquardt, H. and Sternberg, S. S. 1975. Some observations on the toxicity of adriamycin (NSC123127). Cancer Chemother. Rep. 6: 177-181.

17. Rojkind, M., Giambrone, M. A. and Biempica, L. 1979. Collagen types in normal and cirrhotic liver. Gastroenterology 76: 710-719.

18. Schnaper, H. W., Kopp, J. B., Poncelet, A. C., Hubchak, S. C., Stetler-Stevenson, W. G., Klotman, P. E. and Kleiman, H. K. 1996. Increased expression of extracellular matrix proteins and decreased expression of matrix protease after serial passage of glomerular mesangial cells. J. Cell Sci. 109: 2521-2528.

19. Tamaki, K., Okuda, S., Ando, T., Iwamoto, T., Nakayama, M., and Fujishima, M. 1994. TGF- $\beta 1$ in glomerulosclerosis and interstitial fibrosis of adriamycin nephropathy. Kidney Int. 45: $525-536$.

20. Tamura, K., Manabe, N., Uchio, K., Miyamoto, M., Kinoshita, A., Ogura, A., Yamamoto, Y., Nagano, N., Furuya, Y. and Miyamoto, H. 2000. Characteristic changes in carbohydrate profile in the kidneys of hereditary nephrotic mice (ICGN strain). J. Vet. Med. Sci. 62: 379-390.

21. Tomooka, S., Border, W. A., Marshall, B. C. and Noble, N. A. 1992. Glomerular matrix accumulation is linked to inhibition of the plasmin protease system. Kidney Int. 42: 1462-1469.

22. Uchio, K., Manabe, N., Kinoshita, A., Tamura, K., Miyamoto, M., Ogura, A., Yamamoto, Y. and Miyamoto, H. 1999. Abnormalities of extracellular matrices and transforming growth factor $\beta 1$ localization in the kidney of the hereditary nephrotic mice (ICGN Strain). J. Vet. Med. Sci. 61: 769-776.

23. Uchio, K., Manabe, N., Tamura, K., Miyamoto, M., Ogura, A., Yamamoto, Y. and Miyamoto, H. 2000. Decreased activities of matrix metalloproteinases in the kidneys of hereditary nephrotic mice (ICGN Strain). Nephron 86: 145-151.

24. Weening, J. J. and Rennke, H. G. 1983. Glomerular permeability and polyanion in adriamycin nephrosis in the rat. 1983. Kidney Int. 24: 152-159. 